\title{
The expected value of perfect information in unrepeatable decision-making
}

\author{
Mercedes Boncompte Pons \\ Department of Economic, Financial and Actuarial Mathematics \\ Universidad de Barcelona, 08034 Barcelona, Spain
}

\begin{abstract}
This paper reflects on the concept of the "Expected Value of Perfect Information" (EVPI) and the procedure used to determine it. It is widely accepted that this value is the difference between the expected value when we have perfect information and the best expected value provided by alternatives. However, this difference often results in values that no rational decision-maker would accept. Here, we overcome this difficulty by defining the "Value of Perfect Information for the Problem" (VPIP) where we consider not only the price of perfect information $(E V P I)$ but also two additional parameters: the "Loss to be Avoided" and "The Most Favourable Payoff in the Worst Scenario". In this way, we are able to obtain a more accurate value of the amount a decision-maker might be willing to pay for perfect information. We also seek to show that the indiscriminate employment of probability theory, based by definition on the repetition of the experiment, can be misleading in the case of decisions which, owing to the very nature of the problem, are unrepeatable. Keywords: Expected Value of Perfect Information, Decision Theory, Probability Theory, Opportunity Cost, Risk Aversion, Unrepeatable Decision
\end{abstract}

\section{Introduction}

The "Expected Value of Perfect Information" (EVPI) was defined by Szaniawski in 1967 as "the highest price the decision-maker would be prepared to pay for perfect information" ${ }^{1}$. The study of perfect information and the amount a decision-maker might be willing to pay for it was subsequently developed on the basis of this article. Szaniawski only discusses finite decision problems where both the set of alternatives and

Email address: mboncompte@ub.edu (Mercedes Boncompte Pons)

${ }^{1}$ Szaniawski (1967), p. 412 
the set of states of nature are finite. Moreover, he points out that the value of perfect information depends on the decision criterion used by the decision-maker. Szaniawski also gives exact formula for the Maximin criteria, Laplace, Minimax loss, Hurwicz, $\beta$-criterion and maximizing expected utility.

When discussing maximizing expected utility, the expected value of perfect information is calculated, following Szaniawski's formula, as the difference between the expected value when we have perfect information and the best expected value provided by alternatives ${ }^{2}$. Furthermore, he demonstrated that the EVPI matches the minimum expected cost of opportunity. Hereinafter, all books and articles on decision theory have used this formula to calculate the EVPI.

In the related literature, various decision problems have shown that the mechanical application of this formula can lead to surprising outcomes. In the present paper, we overcome this difficulty by defining the "Value of Perfect Information for the Problem" (VPIP), in which we consider not only the price of perfect information ( $E V P I)$ established by Szaniawski but two additional parameters: the "Loss to be Avoided" and "The Most Favourable Payoff in the Worst Scenario". This first parameter is a key concept to ensure the correct evaluation of the value of perfect information in each specific problem.

The idea for this paper originates from the contrast between theoretical results and the results that can be intuited from practical problems. For this reason, it draws on examples that can facilitate reasoning, yet without sacrificing mathematical accuracy.

Additionally, we consider the relationship between probability theory and decision theory, taking into account that the former is sustained by the repetition of experiments and that the latter needs to be right when making unrepeatable decisions.

We focus on finite decision problems, that is, problems where both sets of possible actions and states of nature are finite. We structure our contribution as follows:

Section 2 recognises the need to review the concept and the calculation of EVPI by addressing the oil-drilling problem, a classic in decision theory. It is this which has given rise to the present paper and which serves as its unifying thread. Section 3 reviews some of the many contributions on EVPI. We should

\footnotetext{
${ }^{2}$ Szaniawski (1967), p. 421
} 
stress, nonetheless, that in the theoretical field continuous variables have always been used. As a result, the formula given by Szaniawski in 1967 has retained full validity in practical finite decision problems. Section 4 examines the relationship between probability theory and decision theory. In section 5 , we summarise what is known about EVPI in finite problems. Sections 6 and 7 are the core of this paper. Section 6 introduces the concept of the "Loss to be Avoided" $(L)$, which is the primary motive driving the decision-maker to pay for perfect information. In this section, we present a general formula for deducing this $L$ value. We derive this formula by applying an inductive reasoning to a further two examples. Section 7 defines a new upper bound for the value of perfect information for each specific problem, which we name the "Value of Perfect Information for the Problem" (VPIP). In this section, we present the three requirements that the VPIP must fulfil. Then, we solve the three examples presented by taking these requirements into consideration. This section also considers the impact that obtaining perfect information has on various decision problems. Section 8 analyses an additional problem (Bierman et al., 1994) which gives a surprising result, unless the $V P I P$ is used. Finally, section 9 presents our conclusions.

\section{The need to review the concept of EVPI and its calculation}

The need to review the concept of EVPI and its calculation becomes evident when analysing the results of various published problems. Specifically, the decision problem that has triggered this article is a classic one in decision theory: the oil-drilling problem (Hammond, 1967). The EVPI proposed therein is surprising and, at least on initial inspection, unacceptable. In this article, we reflect on the EVPI concept and examine its characteristics as well as its application to different problems.

We use the updated version of the problem (Hillier and Lieberman, 2010):

The GOFERBROKE COMPANY owns a tract of land that may contain oil. A consulting geologist has reported to management that he believes there is one-in-four chance of striking oil. Because of this prospect, another oil company has offered to purchase the land for \$90,000. However, Goferbroke is considering holding the land in order to drill for oil itself. The cost of drilling is $\$ 100,000$. If oil is found, the resulting expected revenue will be \$800,000, so the company's expected profit (after deducting the cost of drilling) will 
Table 1: The oil-drilling problem

\begin{tabular}{ccc} 
& Drilling $(\$)$ & Selling $(\$)$ \\
\hline Oil (0.25) & 700,000 & 90,000 \\
Dry (0.75) & $-100,000$ & 90,000 \\
\hline
\end{tabular}

be $\$ 700,000$. A loss of $\$ 100,000$ (the drilling cost) will be incurred if the land is dry (no oil).

We propose the following questions:

1. What decision should the company's manager take if he is risk neutral and the utility function is $U(x)=x ?$

2. The manager believes that the geological study is not sufficient and requests a seismological study. If the study was fully reliable, how much would the manager be willing to pay for it?

\section{Solution:}

Table 1 provides a summary of the problem.

1. We calculate:

Expected value (drilling)

$=700,000 * 0.25-100,000 * 0.75=100,000$

Expected value (selling) $=90,000$.

Therefore, the manager will drill based on the fact that drilling, as opposed to selling, provides a higher expected value.

2. The manager will be willing to pay the EVPI, in other words, the difference between the expected value that he would obtain if he had perfect information and the best expected value offered by the various alternatives.

In our problem, if we knew we would strike oil, then we would drill; an outcome that will occur in $25 \%$ of cases. In other contingencies, we would sell (75\%). Based on the value we would expect to obtain with these amounts, we would subtract the expected value of drilling, which is the option we would choose if we did not have perfect information. 
EVPI

$=(700,000 * 0.25+90,000 * 0.75)-100,000$

$=142,500$

Answer: The manager would be willing to pay up to $\$ 142,500$ for perfect information.

The aim of this paper is to highlight that in many instances the EVPI obtained is unacceptable for a sensible decision-maker.

Here, the company is unlikely to be willing to pay $\$ 142,500$ for a seismic study to obtain perfect information, since this value exceeds the cost of drilling, which gives the perfect information par excellence. Indeed, the reason why the manager resorts to decision theory is the cost involved in drilling. If drilling was cost free, no one would consider selling without drilling ${ }^{3}$.

\section{Related Literature}

The study of the amount of money a decision-maker is willing to pay to have perfect information when making his decision has triggered a broad literature since Szaniawski first published his article, "The value of perfect information" (1967), in which he presents the formula that we continue to use to this day. Szaniawski, however, only discusses finite decision problems. We would like to stress that practically everything that has been written in the theoretical field about the value of perfect information uses continuous distribution functions. Below, we present various examples of such studies undertaken in several areas.

The EVPI has been studied from many different points of view: using optimal control theory (Mehrez, 1985b), analysing the additivity of EVPIs when the perfect information comes from several sources (Samson et al., 1989), or calculating EVPIs through influence diagrams (Zhang et al., 1993). It has also been shown

\footnotetext{
${ }^{3}$ It might be thought that this paradox is due to the decision-maker's attitude to risk. As is known, decision theory reflects the decision-maker's aversion or predisposition to risk by choosing concave or convex utility functions, respectively. However, using a concave function as a decision-maker's utility function does not ensure that the EVPI will be acceptable. For instance, in the oil-drilling problem, using $U(x)=\ln (x+100,001)$, we obtain $E V P I=82,147$ (Lawrence, 1999); but using $U(x)=\sqrt{x+100,000}$, we obtain $E V P I=113,074$, which is still higher than the cost of drilling.
} 
that the value of information will sometimes be negative for an agent who violates the independence axiom of expected utility theory (Wakker, 1988). However, the value of perfect information will always be nonnegative if the agent satisfies a weak dominance axiom (Schlee, 1991). Thon and Thorlund-Petersen(1993) studied the sufficient conditions on the utility function and the nature of the change in riskiness for the value of information to increase; additionally, they related the results obtained with the maximum value of such information. In 1999, Hammitt and Shlyakhter studied the relation between the expected value of information and the prior distribution used to represent current information. Other studies have identified bounds on the maximum amount that the decision-maker would be willing to pay for the perfect information (Huang et al., 1977; Mehrez, 1985a).

Many applications have been made in different branches of science and technology, including agriculture (Meza et al., 2003); medicine (Felli and Hazen, 1998; Welton et al., 2011; Briggs et al., 2012) ; pharmacy (McCullagh et al., 2016); and engineering (Chazarra et al., 2017; Le et al., 2014). This last article forms part of an interesting monograph about the value of information that includes numerous other references (Keisler, 2014).

\section{Decision Theory and Probability Theory}

Decision theory is an interdisciplinary field of study that analyses how somebody chooses a particular course of action, from a set of different possibilities, which leads to the best result, while respecting that person's preferences (González, 2004).

Various criteria can be used to solve a decision problem $^{4}$ and choosing the one that best reflects the decision-maker's intention is the first decision that has to be taken since, as we know, the outcomes are likely to differ depending on the criteria used (Laporte and Ouellet, 1980).

\footnotetext{
${ }^{4}$ We mention some of the main ones: Maximax (which chooses the alternative that offers the best consequence), Maximin (which chooses the alternative that offers the best of the worst consequences), Laplace (which considers all states of nature as having the same probability and chooses the alternative that has the best expected value), Savage (which chooses the alternative that guarantees the minimum opportunity cost), and Bayes (which calculates the probability of every state of nature and chooses the alternative with the best expected value).
} 
Mathematics, via probability theory, sheds light on decision theory and is a basic tool for its develop$\operatorname{ment}^{5}$

The examples presented in books on decision theory concern "important" decisions, either financially, because of the amount of money involved, or socially, because of the consequences for the decision-maker's private or public life. A fairly common feature of these decisions is their "irreversibility". Indeed, decisions are often irreversible, as an about-face implies a serious financial problem or having to address difficulties of another nature. They are, as such, "unique" decisions, that is, those taken rarely, when electing, for example, to invest or not in a particular project, or to change or to stay in a job. We are not dealing with decisions that are easily made or, at least, decision theory has not been developed for decisions of this kind.

Probability theory is based on the study of the results obtained after repeating the same experiment. The more the experiment is repeated, the greater is our precision in establishing the probability that we will obtain a certain result again. However, from both theoretical and practical points of view, considerable controversy is attached to the notion of the "repetition of experiments" (Robert, 2001).

When decision-makers use probability theory to determine the expected value of their payoff (positive or negative) and opt for a certain alternative, they should not forget that what they have is merely an indicator or, if you will, a fictitious value that only serves as a point of comparison with other indicators, or expected values, that result from opting for other alternatives. It can never be considered a real value.

In this article, we identify various aspects about which a decision-maker must exercise caution when using the expected utility model to calculate the $E V P I^{6}$.

If we return to the oil-drilling problem outlined in the Introduction, the claim that the expected value is 100,000 when the decision-maker opts to drill serves merely to compare this course of action with the

\footnotetext{
${ }^{5}$ However, there are many articles that, for various reasons, question the standard decision theory -namely, the expected utility model- and propose alternative models such as the "unexpected utility" or the "nonlinear expected utility" (MacCrimmon and Larsson, 1979; Machina, 1987; Lawrence, 1999).

${ }^{6}$ Similarly, although in another context, Ekenberg et al. (1997) point out that "while a certain evaluation of a strategy may result in an acceptable expected utility, the consequences of adopting it might be so dire that it should nevertheless be avoided" and suggest establishing certain security constraints.
} 
expected value of the option of selling, valued at 90,000. However, if the decision-maker drills, under no set of circumstances will his profit be $\$ 100,000$ : either he will make a profit of $\$ 700,000$ or he will run up a loss of $\$ 100,000$. Only if the decision-maker has the opportunity to make this decision thousands of times, by earning $\$ 700,000$ a quarter of the times and losing $\$ 100,000$ on all other occasions, would he end up with a balance of $\$ 100,000$, as the expected value predicts. ${ }^{7}$ Clearly, the difficulty in taking the decision lies precisely in the fact that it can only be taken once.

We believe that the decision-maker must be especially cautious when using probability theory to make decisions. For example, if we compare the expected value of drilling $(100,000)$ with that of selling $(90,000)$, decision theory advises us to drill because the expected value of this action is higher. It is our contention that a more accurate reading would set out the resolution in negative terms, intimating that decision theory, in accordance with probability theory, "does not discourage him" from drilling. If, however, the expected value of drilling had been lower than the expected market value, probability theory would indicate to us that statistically he would be ill-advised to drill.

Given that the theory of probability does not discourage us from drilling, it is perfectly consistent on the manager's part to ask for a seismological study in order to increase his degree of certainty.

In problem solving, the decision-maker should not forget that real data are constantly being mixed with probabilistic data and that a superficial reading of the decision tree could lead to unfortunate conclusions being drawn.

\section{The Expected Value of Perfect information (EVPI)}

In decision theory, the EVPI is defined as the price an individual would be willing to pay to have access to perfect information (Szaniawski, 1967; Hubbard, 2014).

\footnotetext{
${ }^{7}$ Note that in the case of selling, the expected value matches the actual value since, if you sell, the probability of obtaining $\$ 90,000$ is $100 \%$.
} 


\subsection{The EVPI is an upper bound}

We understand that the EVPI always provides an "upper bound" for the potential value of any experiment we can undertake to improve our information (Hillier and Lieberman, 2010; López Cachero, 1989).

Mathematically, the upper bound of a set $A$ is defined as follows:

Be $A \subset \mathbb{R}, a \in \mathbb{R}$

$a$ is an upper bound of the set $A \Leftrightarrow a \geq x \forall x \in A$

When an upper bound $a$ belongs to $A$, then $a$ is called a maximum. Clearly, a maximum gives us more accurate information about the set $A$ than an upper bound.

But the maximum of the set of amounts that a decision-maker would be willing to pay to have perfect information cannot be objective, in the sense that it would probably not result from the application of a formula but would depend on the decision-maker himself and on his aversion/predisposition to risk.

For this reason, it could be useful to specify an upper bound as established by the EVPI formula. However, we should stress that this upper bound may lie far beyond the maximum that a sensible decisionmaker would be willing to pay in order to have perfect information.

While it is true that the price of perfect information cannot be anything other than the difference between the expected value we obtain from having this information and the expected value without it, decision theory is unable to inform the decision-maker that this value is the maximum he should pay for perfect information, regardless of anything else. In the same way, the correct calculation of the price of a product does not justify its purchase.

\subsection{Expected Value of Perfect Information and Opportunity Cost of the Best Alternative}

Table 2 lists the notations that we will use in our discussion.

First, in order to clarify the following explanation, we redefine the "Expected Value of Perfect Information" $(E V P I)$ as the price of perfect information. We calculate this in the usual manner: that is, as the difference between the expected value that we obtain with perfect information and the best expected value without perfect information. 
Table 2: Notations

\begin{tabular}{ll}
$A_{j}$ & The alternatives $(1 \leq j \leq n)$ \\
$S_{i}$ & The scenarios $(1 \leq i \leq m)$ \\
$p_{i}$ & The probability of $S_{i}$ \\
$E\left(A_{j}\right)$ & The expected value of $A_{j}$ \\
$x_{i j}$ & The payoff with $A_{j}$ in $S_{i}(1 \leq i \leq m, 1 \leq j \leq n)$ \\
$\tilde{x}_{i}$ & The best value in $S_{i}(1 \leq i \leq m)$ \\
$A_{\tilde{j}}$ & The alternative with the best expected value \\
$C o_{i j}$ & The opportunity cost of $A_{j}$ in $S_{i}(1 \leq i \leq m, 1 \leq j \leq n)$ \\
$S_{\underline{i}}$ & The worst scenario (where $A_{\tilde{j}}$ obtains its worst result) \\
$x_{i \tilde{j}}$ & The worst payoff of the best alternative $A_{\tilde{j}}$ \\
$L$ & The Loss to be Avoided \\
$P_{c}$ & The payment pertaining to the decision-maker in the current moment \\
$F P W$ & The Most Favourable Payoff in the Worst Scenario \\
$E V P I$ & The Expected Value of Perfect Information \\
$V P I P$ & The Value of Perfect Information for the Problem \\
$V P I P_{R}$ & The R-Value of Perfect Information for the Problem \\
\hline
\end{tabular}


That is, let a decision problem $P$ with $n$ alternatives $A_{j}(1 \leq j \leq n)$ and $m$ scenarios $S_{i}(1 \leq i \leq m)$ each have a probability $p_{i}(1 \leq i \leq m)$.

Let $x_{i j}(1 \leq i \leq m, 1 \leq j \leq n)$ be the payoff that the decision-maker obtains if he chooses alternative $A_{j}$ and scenario $S_{i}$ occurs. Hereinafter, we assume that the decision is about maximizing benefits or profits; however, in this paper, we will always refer to a "better" payoff rather than to a "greater" payoff, in order to include the problems about minimizing costs. In this cases, it is sufficient to replace "max" with "min".

Let $\tilde{x}_{i}(1 \leq i \leq m)$ be the best value or the highest profit associated with each scenario, that is:

$$
\tilde{x}_{i}=\max _{j} x_{i j}
$$

Let $E\left(A_{j}\right)$ be the expected value of $A_{j}$.

Let $A_{\tilde{j}}$ be the best alternative, that is, the alternative with the best expected value:

$$
E\left(A_{\tilde{j}}\right)=\max _{j} E\left(A_{j}\right)=\max _{j} \sum_{i=1}^{m} x_{i j} p_{i}=\sum_{i=1}^{m} x_{i \tilde{j}} p_{i}
$$

Therefore,

$$
E V P I=\sum_{i=1}^{m} \tilde{x}_{i} p_{i}-\sum_{i=1}^{m} x_{i \tilde{j}} p_{i}
$$

Moreover, if, as per usual, we refer to the opportunity cost $\left(C o_{i j}\right)$ of the alternative $A_{j}$ in scenario $S_{i}$ as the difference between the best payoff in scenario $S_{i}$ and the payoff that takes place with alternative $A_{j}$ in scenario $S_{i}$ :

$$
C o_{i j}=\tilde{x}_{i}-x_{i j}
$$

then, we obtain that the EVPI can also be interpreted as the expected value of the opportunity cost of the best alternative, that is, the expected value of the amount that we lost with the best alternative with regard to the best option in each scenario (Szaniawski, 1967; Laporte and Ouellet, 1980):

$$
E V P I=\sum_{i=1}^{m} \tilde{x}_{i} p_{i}-\sum_{i=1}^{m} x_{i \tilde{j}} p_{i}=\sum_{i=1}^{m}\left(\tilde{x}_{i}-x_{i \tilde{j}}\right) p_{i}
$$

Furthermore, since seeking the alternative that minimizes the expected value of the opportunity cost coincides with seeking the alternative that maximizes the expected value, then the EVPI matches the 
minimum expected opportunity cost (Szaniawski, 1967; Laporte and Ouellet, 1980), that is:

$$
\begin{aligned}
\min _{j} E\left(C o_{i j}\right) & =\min _{j} \sum_{i=1}^{m}\left(\tilde{x}_{i}-x_{i j}\right) p_{i} \\
& =\min _{j}\left[\sum_{i=1}^{m} \tilde{x}_{i} p_{i}-\sum_{i=1}^{m} x_{i j} p_{i}\right] \\
& =\sum_{i=1}^{m} \tilde{x}_{i} p_{i}-\max _{j} \sum_{i=1}^{m} x_{i j} p_{i} \\
& =\sum_{i=1}^{m} \tilde{x}_{i} p_{i}-\sum_{i=1}^{m} x_{i \tilde{j}} p_{i} \\
& =E V P I
\end{aligned}
$$

Finally, note that an upper bound of the EVPI is $\max _{i}\left(\tilde{x}_{i}-x_{i \tilde{j}}\right)$ :

$$
\begin{aligned}
E V P I & =\sum_{i=1}^{m}\left(\tilde{x}_{i}-x_{i \tilde{j}}\right) p_{i} \\
& \leq \max _{i}\left(\tilde{x}_{i}-x_{i \tilde{j}}\right) \sum_{i=1}^{m} p_{i} \\
& \leq \max _{i}\left(\tilde{x}_{i}-x_{i \tilde{j}}\right)
\end{aligned}
$$

which means that the EVPI is upper bounded by the highest opportunity cost that results in the best alternative $A_{\tilde{j}}$ in certain scenarios.

In the next section, we introduce a new concept, which we believe should be taken into account when measuring the value of perfect information.

\section{The Loss to be Avoided ( $L$ )}

We understand that a decision-maker is willing to pay for perfect information because he wants to avoid running up a loss. For example, in the oil-drilling problem, the highest loss that he can make is $\$ 100,000$. Clearly, therefore, the main objective of any study that provides us with perfect information is to prevent such a loss. Below, we discuss whether it is possible to define the concept of the "Loss to be Avoided" $(L)$ generally for all problems.

Obviously, the decision problem would no longer be difficult if the best alternative $A_{\tilde{j}}$ was the best in all scenarios. However, it is possible that there is a scenario $S_{\underline{i}}$ where the best alternative offers a worse result than other alternatives. It is in this scenario where the decision problem lies. 
Definition 1. The scenario where the best alternative $A_{\tilde{j}}$ gives its worst result is defined as the "worst scenario for the best alternative" or simply "the worst scenario" and we denote it as $S_{\underline{i}}$, that is:

$$
\min _{i} x_{i \tilde{j}}=x_{\underline{i} \tilde{j}}
$$

It does not seem difficult to determine what the worst scenario is, since it is the one of greatest concern to the decision-maker. In the oil-drilling problem, the worst scenario would clearly be that in which they fail to strike oil.

Therefore, we understand that choosing the best alternative can easily entail the risk of making an important loss in one or more scenarios, and it is precisely this loss that we want to avoid when we resort to decision theory and we analyse the EVPI.

The next issue is how to identify the "Loss to be Avoided". We begin by seeking to determine whether we can identify $L$ with one of the concepts that we enumerate below. Then, by analysing two new problems, we discard these concepts in order to obtain the final formulation. To present these concepts, once again, we draw on the oil-drilling problem.

In the oil-drilling problem, the "Loss to be Avoided" is, at least at first sight, the drilling costs, which is the minimum payment offered by the best alternative. It might be thought that this is always the case. It might also be thought that we should take into account the $\$ 90,000$ profit that we lose when we do not take up the option of selling, which is the best alternative in the worst scenario. That is, we might also consider $L$ to coincide with the opportunity cost of the best alternative in the worst scenario or, maybe, that $L$ coincides with the highest opportunity cost that can be obtained with the best alternative but perhaps not in the worst scenario.

Therefore, we need to ask ourselves:

A. Is $L$ the highest opportunity cost that can be obtained with the best alternative $A_{\tilde{j}}$ ?

B. Is $L$ the opportunity cost associated with the best alternative $A_{\tilde{j}}$ in the worst scenario $S_{\underline{i}}$ ?

C. Is $L$ the minimum payment associated with the best alternative $A_{\tilde{j}}$, that is, the payment associated with the best alternative in the worst scenario $\left(x_{\underline{i} \tilde{j}}\right) ?$ 
Table 3: The horse-racing problem ${ }^{a}$

\begin{tabular}{lcccc}
\hline & A1 & A2 & A3 & $\tilde{x}_{i}$ \\
\hline Lady (0.5) & -30 & $\mathbf{2 0}$ & 15 & 20 \\
Fury (0.2) & 10 & $\mathbf{5 0}$ & 25 & 50 \\
Black (0.3) & $\mathbf{1 3}$ & -50 & 0 & 13 \\
$E\left(A_{j}\right)$ & -9.1 & 5 & $\mathbf{1 2 . 5}$ & \\
\hline
\end{tabular}

${ }^{a}$ The last row presents the expected values of each game of chance and the last column shows the highest payment that is obtained by each winning horse in some of the games.

To answer these questions, let us consider two additional decision problems.

- First problem: The horse-racing problem

A player is offered the opportunity to participate in one of the following games of chance (A1, A2 and A3). The payments he receives will depend on which horse wins a certain race: Lady is the favourite in $50 \%$ of the betting. Fury is favourite in $20 \%$ and Black in $30 \%$.

The problem is presented in Table 3.

$$
\begin{aligned}
E V P I & =(20 * 0.5+50 * 0.2+13 * 0.3)-12.5 \\
& =23.9-12.5=11.4
\end{aligned}
$$

The best expected value criteria indicate choosing game A3 because its expected value is 12.5 , which is clearly superior to the values of the other two games. It is also clear that the worst scenario in A3 is that Black wins the race. This is the scenario that is likely to cast doubt in the mind of the decision-maker, since if he had perfect information and knew that Black would win, he would choose game A1.

In all probability, the decision-maker would indeed choose A3, because the expected value is the highest and because in this game he "cannot lose anything".

What, therefore, would be the "Loss to be Avoided" value in this game?

In Table 4 we write the associated opportunity costs. 
Table 4: The horse-racing problem. Opportunity costs

\begin{tabular}{cccc} 
Opp. costs & A1 & A2 & A3 \\
\hline Lady (0.5) & 50 & $\mathbf{0}$ & 5 \\
Fury (0.2) & 40 & $\mathbf{0}$ & 25 \\
Black (0.3) & $\mathbf{0}$ & 63 & 13 \\
\hline
\end{tabular}

A. Is the "Loss to be Avoided" the highest opportunity cost associated with A3, that is, 25?

We believe that the decision-maker cannot perceive as a loss what he would "fail to win" if Fury were to win the race, because this is precisely the decision-maker's most desired scenario (who after all chose A3), since in this game he obtains the highest profit.

Note, therefore, that the opportunity cost depends on the profits associated, perhaps with a very low probability, with other alternatives and that these amounts could easily constitute "a false promise".

B. Is the "Loss to be Avoided" the opportunity cost associated with A3 if Black wins, that is, 13 ?

We believe that if Black wins, the decision-maker will consider himself unlucky as he has neither won or lost anything. Moreover, he will be glad that he did not choose A2. However, we believe that he cannot perceive 13 as a "loss" as it was a prize that he had not yet won.

C. Is the "Loss to be Avoided" the minimum payment associated with the best alternative A3, that is, $0 ?$

Indeed, we believe that the decision-maker will consider that in the worst scenario provided by game A3, he has lost nothing.

Therefore, in this decision problem, the "Loss to be Avoided" value is 0 .

Let us further our explanation by examining a second problem.

- Second problem: The airline problem

(French, 1986)

Consider the case of an airline that has the opportunity to buy a second-hand airplane. The overall payoff for the airline depends on whether the aircraft turns out to be very reliable, moderately reliable or 
Table 5: The airline problem

\begin{tabular}{cccc}
\hline Reliability & Buying & Not buying & $\tilde{x}_{i}$ \\
\hline High (0.2) & $\mathbf{1 0 0}$ & 17 & 100 \\
Mod. (0.3) & $\mathbf{3 4}$ & 17 & 34 \\
No (0.5) & 1 & $\mathbf{1 7}$ & 17 \\
$E\left(A_{j}\right)$ & $\mathbf{3 0 . 7}$ & 17 & \\
\hline Table 6: The airline problem. Opportunity costs \\
\hline Reliability/Opp. costs & Buying & Not buying \\
\hline High $(0.2)$ & & $\mathbf{0}$ & 83 \\
Mod. $(0.3)$ & & $\mathbf{0}$ & 17 \\
No (0.5) & & 16 & $\mathbf{0}$ \\
\hline
\end{tabular}

unreliable after economically evaluating customer satisfaction or dissatisfaction, the economic income, the start-up and maintenance costs, etc. This payoff is reflected in Table 5.

The payoff is expressed in million $\$$. If the aircraft is not purchased, we assume the payoff will be the same as in the initial situation.

In this problem, the expected value criterion indicates that the best alternative is "To buy the airplane". Obviously, the worst scenario is the one in which the aircraft turns out to be "Unreliable" because then the company fails to improve its real profit of $\$ 17$ million, and the benefits are reduced to $\$ 1$ million.

What, therefore, would be the "Loss to be Avoided" value in this problem?

In Table 6 we write the associated opportunity costs.

In this problem the "Loss to be Avoided" is the difference between the current profit, on which the company can supposedly count if it does not take the decision to buy the airplane (that is, $\$ 17$ million), and the profit that it can obtain if it opts for the best alternative and finds itself in the worst scenario (that is, $\$ 1$ million). Clearly, what might hold the company back in its decision to buy the aircraft is the possibility of finding itself in the worst scenario and "losing" \$16 million. 
Table 7: The airline problem. First variant

\begin{tabular}{lccc}
\hline Reliability & Buying & Not buying & $\tilde{x}_{i}$ \\
\hline High (0.2) & $\mathbf{1 0 0}$ & 17 & 100 \\
Mod. (0.3) & $\mathbf{3 4}$ & 17 & 34 \\
No $(0.5)$ & -1 & $\mathbf{1 7}$ & 17 \\
$E\left(A_{j}\right)$ & $\mathbf{2 9 . 7}$ & 17 & \\
\hline
\end{tabular}

Thus, in this case, the "Loss to be Avoided" is the opportunity cost associated with the best alternative in the worst scenario. Unlike the horse-racing problem, the "Loss to be Avoided" is not the minimum payment associated with the best alternative but rather the opportunity cost associated with the best alternative in the worst scenario. Note that were we to modify the problem slightly, so that the associated payment in the worst scenario was negative, for instance, if the aircraft turned out to be unreliable, then would be a loss of $\$ 1$ million, and so the "Loss to be Avoided" would still be the opportunity cost associated with the best alternative in the worst scenario, that is, $\$ 18$ million. (see Table 7 ).

Why does this difference exist between the horse-racing problem, where the loss coincides with the payment associated with the best alternative in the worst scenario, and the airline problem, where the loss is the opportunity cost associated with the best alternative in the worst scenario? We believe that the key lies in the time reference. In the first problem, we start from a present moment in which the current profit is $\$ 0$, whereas in the airline problem, in the present moment we have $\$ 17$ million.

Let us suppose that in the airline problem a third alternative exists, namely acting as intermediaries, in other words, buying the aircraft and then immediately reselling it. Additionally, let us also suppose that the payments are not strictly the profit but rather the result of a utility function. In this case, if the airplane proved to be very reliable, the outcome would be understood to be a $\$ 10$ million loss because we would have sold a good plane that could have yielded greater profits for the company. On the other hand, if it proved to be unreliable, our utility would be $\$ 20$ million. The new problem is presented in Table 8 .

Now, buying the aircraft is still the best alternative and the Loss to be Avoided is still $\$ 18$ million, which 
Table 8: The airline problem. Second variant

\begin{tabular}{lcccc} 
Reliability & Buying & Not buying & Inter. & $\tilde{x}_{i}$ \\
\hline High (0.2) & $\mathbf{1 0 0}$ & 17 & -10 & 100 \\
Mod. (0.3) & $\mathbf{3 4}$ & 17 & 15 & 34 \\
No $(0.5)$ & -1 & 17 & $\mathbf{2 0}$ & 20 \\
$E\left(A_{j}\right)$ & $\mathbf{2 9 . 7}$ & 17 & 12.5 & \\
\hline
\end{tabular}

Table 9: The oil-drilling problem. Opportunity costs

\begin{tabular}{ccc} 
Opp. costs & Drilling & Selling \\
\hline Oil $(0.25)$ & $\mathbf{0}$ & 610,000 \\
Dry $(0.75)$ & 190,000 & $\mathbf{0}$ \\
\hline
\end{tabular}

is neither the minimum payment associated with the best alternative nor the opportunity cost of the best alternative in the worst scenario, which in this instance would be $\$ 21$ million.

Therefore, we are now in a position to define the "Loss to be Avoided".

Definition 2. The "Loss to be Avoided" $(L)$ is the difference between the payment pertaining to the decisionmaker in the present moment $\left(P_{c}\right)$ and the worst payment associated with the best alternative $\left(x_{\underline{i} \tilde{j}}\right)$, provided that this difference is positive; otherwise, the "Loss to be Avoided" will be zero.

$$
L=\max \left\{P_{c}-\min _{i} x_{i \tilde{j}}, 0\right\}=\max \left\{P_{c}-x_{\underline{i} \tilde{j}}, 0\right\}
$$

In other words, if the payment associated with the best alternative in the worst scenario is higher than the payment in the present moment, the "Loss to be Avoided" will be null.

Returning now to the oil-drilling problem that initially triggered the discussion addressed herein, Table 9 describes the opportunity costs array associated with the problem. Note that, according to Definition 2 , the challenge of determining $L$ is actually that of determining $P_{c}$. If the decision-maker understands that at the present moment his payment is $\$ 0$, then the "Loss to be Avoided" will be the drilling costs. Only if the decision-maker understands that the sale of the tract of land will raise $\$ 90,000$ will the "Loss to be 
Avoided" be the $\$ 190,000$ indicated by the opportunity cost and, consequently, the decision-maker could be willing to pay $\$ 142,500$ for perfect information.

In this case, we could probably interpret these implications in the opposite sense: that is, if the decisionmaker is unwilling to pay up to $\$ 142,500$ for perfect information, then it is because he does not actually perceive that his "Loss to be Avoided" is $\$ 190,000$.

Here, we should stress once more that we are hardly entitled to refer to the money that we have not yet gained as a "loss" and that the $\$ 100,000$, which inexorably will have to be drawn from the pocket of the decision-maker if he drills, cannot be considered as being on the same level as the $\$ 90,000$ that will probably be pocketed if the sale is finally formalized.

We believe that placing ourselves in the "present" is what allows us to determine whether $L$ is strictly "what is lost" or whether $L$ also extends to what one "fails to win". Of course, there will be many cases in which the "Loss to be Avoided" will be highly debatable and, therefore, the exclusive competence of the decision-maker.

As we have seen in (2), the EVPI contains in its calculation procedure an approximation of our "Loss to be Avoided" concept, in the sense that its value coincides with the expected value of the opportunity cost associated with the best alternative. However, once again, it is an "upper bound" and the "Loss to be Avoided" can be substantially lower.

In the next section, we present a new concept that provides us with the value of perfect information for each particular problem.

\section{The Value of Perfect Information for the Problem (VPIP)}

\subsection{Deduction and formulation of the VPIP}

We redefined the "Expected Value of Perfect Information" (EVPI) then as the price of perfect information. We distinguish this value from the "Value of Perfect Information for the Problem" (VPIP) and define this as an upper bound of the set of all amounts that a decision-maker is willing to pay in order to obtain the perfect information related to the specific conditions of a problem. Thus, we calculate our own 
value of perfect information by relying not only on the EVPI but also on the data provided by the specific problem we address. A similar approach was developed by Wang et al. (2008).

We calculate the EVPI, as per usual, according to (1), as the difference between the expected value we obtain with perfect information and the best expected value without perfect information.

To calculate the VPIP, the new bound we are seeking must fulfil the following three requirements.

- First requirement: Expected Value of Perfect Information (EVPI)

The first condition is that the upper bound of the VPIP must be lower than that of the EVPI, since it makes little sense to spend more money on the study than the amount identified as the price of perfect information by probability theory.

\section{- Second requirement: Loss to be Avoided $(L)$}

The second condition is that the VPIP must be lower than the loss we want to avoid $(L)$. As explained in section 6 , if we are willing to pay for perfect information, it is precisely because we wish to avoid a greater loss, in the same way that no one would pay an insurance premium higher than the price of the object they want to insure ${ }^{8}$.

\section{- Third requirement: The Most Favourable Payoff in the Worst Scenario $(F P W)$}

The VPIP should be lower than the most Favourable Payoff obtained in the Worst scenario $(F P W)$. In this way, we ensure that the cost of the study is covered in all scenarios.

For instance, in the oil-drilling problem, it could be deduced that the maximum amount we would be willing to pay was $\$ 100,000$, since this represents the cost of drilling. Thus, any amount that gives us perfect information for less than $\$ 100,000$ will be a saving. Nevertheless, if, for example, the study costs $\$ 95,000$ and we find the well to be dry, we will have saved $\$ 5,000$ with regard to the drilling costs and we will opt to sell. However, we would be unable to offset the cost of the study by selling the tract of land.

\footnotetext{
${ }^{8}$ Risk analysis is a valuable approach to adopt in studies of this kind (Marques and Berg, 2011).
} 
Thus, we come to our final formulation of the "Value of Perfect Information for the Problem":

Definition 3. Given a finite decision problem, the "Value of Perfect Information for the Problem"(VPIP) is a higher bound of the value that a decision-maker is willing to pay in order to have perfect information. It is calculated as the minimum between the "Expected Value of Perfect Information" (EVPI), the value of the "Loss to be Avoided" (L) and "The Most Favourable Payoff in the Worst Scenario" (FPW).

$$
V P I P=\min \{E V P I, L, F P W\}
$$

A decision-maker with a greater predisposition to risk might disregard the latter requirement and consider a $V P I P_{R}$ bound acceptable, even though the study costs would signify a net loss in the worst scenario.

$$
V P I P_{R}=\min \{E V P I, L\}
$$

We refer to $V P I P_{R}$ as the "R-Value of Perfect Information for the Problem".

It is common practice in decision theory to refer to "risk-neutral" decision-makers, indicating that they are "trusting" of the expected values associated with the various options and that they ignore the negative consequences that may result; but, in reality, very few decision-makers adopt such an attitude (Hammond, 1967). As a matter of fact, it may not even be acceptable among these decision-makers to proceed without their adhering to the requirement of neither exceeding the value of the "Loss to be Avoided" nor the value of "The Most Favourable Payoff in the Worst Scenario". The procedures developed by decision theory can never lead the decision-maker to risk without warning.

Let us stress once again the importance of keeping in mind that the decision we are to take is unrepeatable and unique, and that the difference in expected values can only help us in a negative sense. That is, it can only serve to warn the decision- maker of the fact that even if he has perfect information, probability theory indicates an increase in the expected value, in this case, of $\$ 142,500$ and no higher. Thus, decision theory advises against spending more than $\$ 142,500$ on the study; but, under no circumstances should decision theory convey the idea to the decision-maker that he would be right to invest any amount below $\$ 142,500$ in order to obtain perfect information without further details. 
Table 10: The oil-drilling problem. VPIP and VPIP $P_{R}$ values depending on $L$

\begin{tabular}{lll}
$L=100,000$ & $L=190,000$ \\
\hline$V P I P$ & $\min \{142,500,100,000,90,000\}=90,000$ & $\min \{142,500,190,000,90,000\}=90,000$ \\
$V P I P_{R}$ & $\min \{142,500,100,000\}=100,000$ & $\min \{142,500,190,000\}=142,500$ \\
\hline
\end{tabular}

\subsection{Oil-drilling problem solution}

To conclude the oil-drilling problem, let's imagine the case of a decision-maker who estimates $L=\$ 100,000$ or $L=\$ 190,000$ and one that is willing or not to pay for Perfect Information, the price of which may not be covered by the profit he will obtain in the worst scenario (as shown in Table 10).

The thesis of the present article is that a very high percentage of decision-makers will prefer the $V P I P$ and $L=\$ 100,000$; thus, they will be unwilling to pay more than $\$ 90,000$ for the perfect information for the drilling problem.

In this case,

$$
V P I P \neq E V P I \neq V P I P_{R}
$$

That is, the price that the decision-maker is willing to pay (VPIP or $\left.V P I P_{R}\right)$ is lower than the "Expected Value of Perfect Information" (EVPI).

However, recall that we have defined the $V P I P$ as an upper bound and not as a maximum, given that the decision-maker could still identify other restrictions related to his problem that might reduce even further the amount he is willing to pay for perfect information.

\subsection{Knowledge of perfect information and the decision-making process}

The primary goal of what is assumed to be perfect information is, clearly, to guide the individual towards taking the right decision. Yet, this is another unusual characteristic of the drilling problem, because having perfect information does not allow the company to make a decision. Indeed, whether the company obtains perfect information by paying for a report or it obtains it by drilling, if it strikes oil, it will suffer no inconvenience. However, if the tract of land is dry, it will have asymmetric information and will no longer be able to sell at the initial $\$ 90,000$ price, which was established for the land with possibilities of oil exploitation. 
If the company overlooks this difficulty and sells for $\$ 90,000$ because the buyer, the second agent, does not have the perfect information that it has, then it will be violating a principle of ethical economy.

Thus, we can identify two types of problem:

1. Problems in which the alternatives remain the same after obtaining perfect information (Airline problem).

2. Problems in which the alternatives change or disappear after obtaining perfect information (Oil-drilling problem).

The change in alternatives after obtaining perfect information constitutes a difficulty that can easily arise in problems when there is a second agent that has to make his decision after the initial decision-maker has taken his and to whom the decision-maker is morally obliged to transmit perfect information. This is the case of the oil companies. In contrast, this inconvenience does not exist in the airline problem, since the aircraft supply company has made its decision before our decision-maker has made his.

\subsection{Resolution of the other two problems applying the VPIP}

Let us now solve the two additional problems posed:

- The horse-racing problem

As discussed, the alternative chosen will be A3, because it has the highest expected value: 12.5. That is, probability theory indicates that this is the alternative with which we will obtain the greatest profits and, therefore, we would choose it. We calculate the EVPI according to (1):

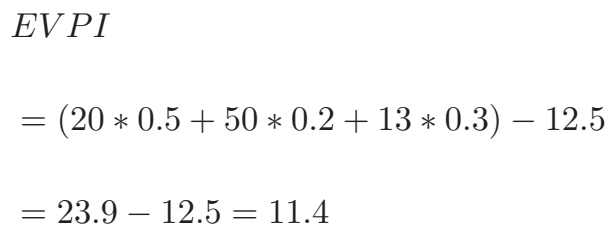

Next, we need to ask ourselves how much we would be willing to pay for the perfect information for this specific problem. The answer is derived from (6):

$$
V P I P=\min \{11.4,0,13\}=0
$$


and it transpires that, as the "Loss to be Avoided" value is zero, we would not be willing to pay anything.

In that case, according to (1), (6) and (7):

$$
V P I P=V P I P_{R} \neq E V P I
$$

- The airline problem

$$
\begin{aligned}
& \text { Expected value (buying) } \\
& \qquad=100 * 0.2+34 * 0.3+1 * 0.5=30.7 \\
& \text { Expected value (not buying) }=17
\end{aligned}
$$

As such, the company would prefer to buy; or, in negative terms, it would not reject the buying option. But how much would the company be willing to pay for perfect information? First, let us calculate the "Expected Value of Perfect Information":

$$
\begin{aligned}
& \text { EVPI } \\
& =(100 * 0.2+34 * 0.3+17 * 0.5)-30.7 \\
& =8
\end{aligned}
$$

We can now compare this value with the "Loss to be Avoided" and the "The Most Favourable Payoff in the Worst Scenario".

As explained, in this problem the "Loss to be Avoided" is $\$ 16$ million. Therefore:

$$
V P I P=\min \{8,16,17\}=8
$$

And in this case,

$$
V P I P=V P I P_{R}=E V P I
$$

In this example, probability theory tells us that having perfect information can provide an incremental benefit of $\$ 8$ million. Moreover, the $V P I P$ and $V P I P_{R}$ bounds certify that this amount is perfectly consistent with the problem, because the loss we want to avoid is much higher and, even in the worst scenario, it would be covered. 
Table 11: Introduction of a new product

\begin{tabular}{lcc}
\hline Demand & Introducing & Not introducing \\
\hline High (0.3) & $\mathbf{4}$ & 0 \\
Low $(0.7)$ & -2 & $\mathbf{0}$ \\
$E\left(A_{j}\right)$ & -0.2 & $\mathbf{0}$ \\
\hline
\end{tabular}

Note also that the definition of $V P I P$ and $V P I P_{R}$ allows the decision-maker to set limits and goals.

In other words, the amount of the "Loss to be Avoided" could be relaxed or otherwise, depending on who the decision-maker is and on his predisposition/ aversion to risk.

\section{An additional conflict resolution problem: introduction of a new product}

The following example, taken from the economic literature (Bierman et al., 1994), also shows that considering only the EVPI can lead to wrong conclusions being drawn.

"It concerns a new product whose potential will be either big or small (that is, the product will be either a success or a failure). The $\$ 4$ million value corresponds to the net profit if its selling potential is big. The loss incurred if the product does not sell well is \$2 million. Table 11 shows the values that condition the decision".

Based on the expected value of each alternative $E\left(A_{j}\right)$, probability theory advises against introducing the new product, and the negative expected value obtained with this option should be sufficient to cause us to abandon the project.

However, if we ask ourselves how much we would be willing to pay for perfect information, we obtain:

$$
\begin{gathered}
E V P I=(4 * 0.3+0 * 0.7)-0=1.2-0=1.2 \\
V P I P=\min \{1.2,0,0\}=0 \\
V P I P_{R}=\min \{1.2,0\}=0
\end{gathered}
$$

Note that even with perfect information the profits in the worst scenario would be 0 . Then, we cannot advise the decision-maker to spend up to $\$ 1.2$ million to obtain perfect information, because in $70 \%$ of the 
cases this money will constitute a net loss. Furthermore, we note that the "Loss to be Avoided" in the chosen alternative is null ${ }^{9}$.

\section{Conclusions}

Important decisions do not accept tests or experiments. As such, the aim of decision theory is to provide the decision-maker with some security when faced with a dilemma that does not accept mistakes, because the price of such mistakes can be very high.

Here, we have stressed that the probability theory is based on the repetition of the experiment, while decision theory focuses essentially on problems that do not allow for any repetition. We propose that the only safe reading of the expected utility of an alternative is in its negative sense. In other words, if we hesitate between two options and the expected utility value of $\mathrm{A}$ is lower than that of $\mathrm{B}$, decision theory, supported by probability theory, will rule out option A and will go on to examine option B using the absolute values that option $B$ entails.

One of the main contributions of this article has been the development of the concept of the "Loss to be avoided" as a key idea for calculating the upper bound of an amount that a decision-maker would be willing to pay in order to have perfect information. The concept of the "Loss to be Avoided" underlies

\footnotetext{
${ }^{9}$ Moreover, underlying the problem there is the idea that the decision-maker hopes to obtain the $\$ 4$ million profit with a 0.3 probability. For this reason, a decision-maker might fail to heed the warning raised by probability theory using the maximum expected utility criterion and, hence, decide to launch the product, with the subsequent risk of losing $\$ 2$ million with a 0.7 probability. In this case, we understand that the "Loss to be Avoided" is the $\$ 2$ million. The calculation can be made as follows:

$$
\begin{gathered}
E V P I=(4 * 0.3+0 * 0.7)-(-0.2)=1.2+0.2=1.4 \\
V P I P=\min \{1.4,2,0\}=0
\end{gathered}
$$

Only in this case, and warning the decision-maker about the risk, we have:

$$
V P I P_{R}=\min \{1.4,2\}=1.4
$$

However, we cannot stress enough that these are upper bounds, because a sensible decision-maker is unlikely to spend $\$ 1.4$ million with a probability of 1 to avoid a loss of $\$ 2$ million with a probability of 0.7 .
} 
the decision-maker's predisposition to pay for perfect information. We have both defined this concept and deduced how it should be calculated in the case of a finite decision problem.

In addition, we have defined a new upper bound for the value that a decision-maker might be willing to pay in order to have perfect information in a particular problem. This new bound, which we call the "Value of Perfect Information for the Problem" (VPIP), takes into account the "Loss to be Avoided" value and another new factor, the "Most Favourable Payoff in the Worst Scenario". These two parameters differ in every problem and allow the VPIP to provide values that are more closely adjusted to reality than those provided by the simple calculation of the EVPI.

We have also formulated a variant of the $V P I P$, the $V P I P_{R}$, which could be acceptable to risk-seeking decision-makers. When these two bounds do not coincide, this indicates to the decision-maker that, in the worst scenario, the $V P I P_{R}$ will not be covered by the payoff he obtains. By introducing these two new bounds, that is, the $V P I P$ and the $V P I P_{R}$, the value of perfect information defined and formulated by Szaniawski can be adapted to each specific decision problem. Moreover, when we analyse the problems that present surprising results in terms of the "Loss to be Avoided" concept and the "Most Favourable Payoff in the Worst Scenario" concept, we obtain more accurate values.

A sensitivity analysis of the results reveals a series of aspects that we would like to address in future research. ${ }^{10}$

\section{Acknowledgments}

I would like to express my warm appreciation to Professor Marina Núñez Oliva, whose contributions have been most valuable in the drafting of this article. I am also deeply grateful to Lijue Lu for her technical support. Finally, we would like to thank the editor-in-chief and the three anonymous reviewers, whose comments and suggestions significantly improved the paper.

\footnotetext{
${ }^{10}$ With continuous distribution functions, see Felli and Hazen (1998).
} 


\section{References}

Bierman, H., Bonini, C. P., Hausman, W. H., Morales Peake, E., 1994. Análisis cuantitativo para la toma de decisiones. Wilmington: Addison-Wesley Iberoamericana.

Briggs, A. H., Weinstein, M. C., Fenwick, E. A., Karnon, J., Sculpher, M. J., Paltiel, A. D., 2012. Model parameter estimation and uncertainty analysis: a report of the ispor-smdm modeling good research practices task force working group-6. Medical Decision Making 32 (5), 722-732.

Chazarra, M., Pérez-Díaz, J. I., García-González, J., 2017. Value of perfect information of spot prices in the joint energy and reserve hourly scheduling of pumped storage plants. Electric Power Systems Research 148, 303-310.

Ekenberg, L., Danielson, M., Boman, M., 1997. Imposing security constraints on agent-based decision support. Decision Support Systems $20(1), 3-15$.

Felli, J. C., Hazen, G. B., 1998. Sensitivity analysis and the expected value of perfect information. Medical Decision Making 18 (1), 95-109.

French, S., 1986. Decision theory: an introduction to the mathematics of rationality. Halsted Press.

González, F. A., 2004. Teoría de la decisión e incertidumbre: modelos normativos y descriptivos. Empiria. Revista de metodología de ciencias sociales (8), 139-160.

Hammitt, J. K., Shlyakhter, A. I., 1999. The expected value of information and the probability of surprise. Risk Analysis 19 (1), 135-152.

Hammond, J. S., 1967. Better decisions with preference theory. Harvard Business Review 45 (November), $123-141$.

Hillier, F., Lieberman, G., 2010. Introduction to operations research. McGraw-Hill Education.

Huang, C. C., Vertinsky, I., Ziemba, W. T., 1977. Sharp bounds on the value of perfect information. Operations Research $25(1), 128-139$

Hubbard, D. W., 2014. How to measure anything: Finding the value of intangibles in business. John Wiley \& Sons.

Keisler, J. M., 2014. Value of information: facilitating targeted information acquisition in decision processes. Environment Systems and Decisions $1(34), 1-2$.

Laporte, G., Ouellet, R., 1980. Théorie de la décision. Montreal: Editions Sciences et Culture Inc.

Lawrence, D. B., 1999. The economic value of information. New York: Springer.

Le, A. H., Tokai, A., Nakakubo, T., 2014. Applying value of information methods to prioritize elements for water quality management with an example of linear alkylbenzene sulfonate in the yodo river, japan. Environment Systems and Decisions $34(1), 110-123$

López Cachero, M., 1989. Análisis y adopción de decisiones. Madrid: Pirámide.

MacCrimmon, K. R., Larsson, S., 1979. Utility theory: Axioms versus paradoxes. In: Expected utility hypotheses and the Allais paradox. Springer, pp. 333-409. 
Machina, M. J., 1987. Choice under uncertainty: Problems solved and unsolved. Journal of Economic Perspectives 1 (1), $121-154$

Marques, R. C., Berg, S., 2011. Risks, contracts, and private-sector participation in infrastructure. Journal of Construction Engineering and Management 137 (11), 925-932.

McCullagh, L., Schmitz, S., Barry, M., Walsh, C., 2016. Non-parametric approach to estimating expected value of perfect information: The usefulness of this approach to a national hta agency. Value in Health 19 (7), A489.

Mehrez, A., 1985a. The effect of risk aversion on the expected value of perfect information. Operations research 33 (2), $455-458$.

Mehrez, A., 1985b. A note on the analysis of the expected value of perfect information with respect to a class of r\&d projects. European journal of operational research $19(2), 217-221$.

Meza, F. J., Wilks, D. S., Riha, S. J., Stedinger, J. R., 2003. Value of perfect forecasts of sea surface temperature anomalies for selected rain-fed agricultural locations of chile. Agricultural and Forest Meteorology 116 (3), $117-135$.

Robert, C., 2001. The Bayesian choice: from decision-theoretic foundations to computational implementation. Springer.

Samson, D., Wirth, A., Rickard, J., 1989. The value of information from multiple sources of uncertainty in decision analysis. European journal of operational research 39 (3), 254-260.

Schlee, E. E., 1991. The value of perfect information in nonlinear utility theory. Theory and Decision 30 (2), $127-131$.

Szaniawski, K., 1967. The value of perfect information. Synthese 17 (4), 408-424.

Thon, D., Thorlund-Petersen, L., 1993. The value of perfect information in a simple investment problem. Information Economics and Policy 5 (1), 51-71.

Wakker, P., 1988. Nonexpected utility as aversion of information. Journal of Behavioral Decision Making 1 (3), $169-175$.

Wang, J., Chaudhury, A., Rao, H. R., 2008. Research notea value-at-risk approach to information security investment. Information Systems Research 19 (1), 106-120.

Welton, N. J., Madan, J., Ades, A. E., 2011. Are head-to-head trials of biologics needed? the role of value of information methods in arthritis research. Rheumatology 50 (suppl_4), iv19-iv25.

Zhang, L., Qi, R., Poole, D., 1993. Incremental computation of the value of perfect information in stepwise-decomposable influence diagrams. In: Proceedings of the Ninth international conference on Uncertainty in artificial intelligence. Morgan Kaufmann Publishers Inc., pp. 400-407. 